\title{
Funciones que caracterizan la gestión de seguridad industrial, ambiente e higiene ocupacional
}

\author{
Functions that characterize the management of industrial safety, environment \\ and occupational hygiene
}

\author{
Yohanny González \\ anny_28_20@hotmail.com \\ Código ORCID: 0000-0002-7132-2037 \\ Universidad del Zulia, Venezuela
}

Artículo recibido septiembre 2019 / Arbitrado en octubre 2019 / Publicado en enero 2020

\begin{abstract}
RESUMEN
Son innumerables las ventajas que representa para cualquier organización contar con la gestión en seguridad industrial, ambiente e higiene ocupacional, es por ello que en este artículo se buscó describir las funciones que la caracterizan en el contexto de las empresas prestadoras de servicio de inspección de equipos estáticos a la industria petrolera. Metodológicamente se tipificó como descriptiva, con diseño de campo, no experimental y transeccional. Para la recolección de datos se utilizó un cuestionario, conformado por 12 ítems con escala de frecuencia. La validez se realizó a través del juicio de expertos, y para calcular su confiabilidad se empleó el método de Alfa de Cronbach, obteniéndose 0,96. La media aritmética se aplicó para el análisis de los datos. Se caracterizó un conjunto de etapas que instrumentan los cursos de acción requeridos por la gestión, en tal sentido, se evidencio moderada presencia en las políticas; organización; planificación y evaluación.
\end{abstract}

Palabras clave: Evaluación, funciones, gestión en seguridad industrial, ambiente e higiene ocupacional, organización, planificación, políticas

\begin{abstract}
There are countless advantages for any organization to have management in industrial safety, environment and occupational hygiene, which is why this article sought to describe the functions that characterize it in the context of companies providing equipment inspection service static to the oil industry. Methodologically it was typified as descriptive, with a field design, not experimental and transectional. For data collection, a questionnaire was used, consisting of 12 items with a frequency scale. Validity was carried out through expert judgment, and the Cronbach's Alpha method was used to calculate its reliability, obtaining 0.96 . The arithmetic mean was applied to analyze the data. A set of stages that implement the courses of action required by management was characterized, in this sense, a moderate presence in policies was evident; organization; planning and evaluation.
\end{abstract}

Key words: Evaluation, functions, industrial safety management, environment and occupational hygiene, organization, planning, policies 


\section{INTRODUCCIÓN}

Toda organización debe mantener entre sus premisas para el éxito de sus negocios el cuidado, en lo que a la seguridad industrial, ambiente e higiene ocupacional se refiere, de todos y cada uno de sus trabajadores, los cuales son el mejor talento y recurso del cual se pueda disponer. En este sentido, es imprescindible brindar a los empleados, sin distinción de sus funciones, bien sean administrativas, obreras $u$ operativas, un puesto de trabajo seguro que sea capaz de prevenir todos aquellos riesgos inherentes a las actividades que en él se desempeñan.

En este contexto, es importante la compatibilidad del ambiente laboral con las capacidades de cada trabajador, la prevención de los accidentes laborales, enfermedades ocupacionales, al fomento y mantenimiento de una cultura de bienestar físico, social, así como mental. De todo lo descrito anteriormente, se desprende el hecho de que en la actualidad muchas organizaciones buscan implantar sistemas de gestión en materia de seguridad industrial, ambiente e higiene ocupacional que le permita no sólo controlar y mitigar los riesgos laborales asociados a sus procesos y actividades, sino además, cumplir con todo lo establecido en el marco legal de cada país, asegurarse de su conformidad de acuerdo a lo establecido en su política de seguridad y salud.

En este marco de ideas, un sistema de gestión de seguridad industrial, ambiente e higiene ocupacional (SIAHO) fomenta los entornos de trabajo seguros, saludables al ofrecer un marco que permite a la organización identificar, controlar coherentemente sus riesgos de salud y seguridad, reducir el potencial de accidentes, apoyar el cumplimiento de las leyes y mejorar el rendimiento en general. Por esta razón, controlar los riesgos de seguridad -salud laboral, asociados a la actividad, no sólo compromete a las empresas a tener un ambiente de trabajo más saludable, sino que les permitirá ser más competitivas.

Es por ello, que en las empresas la gestión de seguridad industrial, ambiente e higiene ocupacional, cada día son más necesarios, como elementos de control para minimizar accidentes o pérdidas en las áreas de trabajo. Así las cosas, para la investigadora, en el ambiente actual, en cumplimiento de este deber, las empresas públicas o privadas, de cualquier sector empresarial, deben realizar la prevención de riesgos laborales mediante la adopción de cuantas medidas sean necesarias para la protección de la seguridad y salud de sus empleados en todos los aspectos. De este modo, desarrollan como estrategia, sistemas de gestión que parte del nivel gerencial para tratar de una forma sistemática los aspectos referidos con el medio ambiente de trabajo.

Al respecto, VanDer (2010), refiere que el objetivo fundamental es la identificación de todos los riesgos asociados con las actividades, la aplicación de procedimiento de trabajo y acciones preventivas para reducir, controlar o eliminar cada uno de esos riesgos presentes en una actividad.

En ese aspecto, la Comunidad Andina de Naciones (CAN) del 26 de septiembre del 2005 involucra los elementos importantes de la gestión de Seguridad Industrial, Ambiente, Ambiente e Higiene (SIAHO), los cuales pueden visualizarse claramente a través del instrumento Andino de Seguridad y Salud en el Trabajo, (propuesto por el reglamento, Resolución 957), vinculante para los cinco países de la subregión Andina: Venezuela, Colombia, Ecuador, Perú y Bolivia. En su artículo 1 recomienda que los 
países miembros lo desarrollen teniendo en cuenta los siguientes aspectos: gestión administrativa, técnica, del talento humano, y procesos operativos básicos.

En el caso de Venezuela, con la entrada en vigencia de la Ley Orgánica de Prevención, Condiciones y Medio Ambiente de Trabajo, (LOPCYMAT, 2005), surge en la legislación laboral venezolana componentes de suma importancia, dado que esta ley tiene como propósito establecer normas y lineamientos que permitan garantizar a los trabajadores y trabajadoras condiciones de seguridad y salud adecuadas en su ambiente de trabajo, lo cual conlleva al ejercicio pleno de sus facultades físicas y mentales mediante la promoción del trabajo seguro y saludable, así como la prevención de los accidentes de trabajo y enfermedades ocupacionales.

Ante este panorama, las empresas del sector de servicios de la industria petrolera nacional, se han dedicado a crear secciones o departamentos en materia de seguridad e higiene, por cuanto son organizaciones en las cuales se realizan actividades industriales con altas posibilidades de ocurrencia de accidentes o exposición a sustancias que, eventualmente, pudieran generar enfermedades profesionales, pues las mismas suponen la manipulación o manejo de fluidos, control de sólidos, labores en laboratorios químicos, almacenamiento de productos químicos, es indudable la necesidad de asegurar las labores en protección de la naturaleza humana y en la continuidad operacional de la organización, factores que inciden de manera significativa en el desempeño de los trabajadores $y$, por ende, en el logro efectivo de los objetivos y metas organizacionales.

En este sentido, estas organizaciones industriales cumplen políticas orientadas a la seguridad industrial, ambiente e higiene ocupacional. No obstante, de acuerdo a conversaciones con personal gerencial de las mismas, es posible que sus sistemas de aseguramiento no estén siendo aplicados de forma adecuada, lo cual parece estar afectando la ocurrencia de situaciones inseguras $y$, en consecuencia, incidiendo de manera directa en el desempeño del personal.

De acuerdo con lo anterior, las gerencias de estas empresas aspiran conocer la realidad y medida de tales efectos en el desempeño, de manera que, al conocerlas, tomar las decisiones pertinentes en cuanto a sus sistemas de gestión de seguridad industrial, ambiente e higiene ocupacional, las cuales les permitan asegurar el logro de sus objetivos y metas de manera eficiente y efectiva.

Bajo esta óptica la investigadora se planteó como objetivo describir las funciones que caracterizan la gestión en seguridad industrial, ambiente e higiene ocupacional en las empresas prestadoras de servicio de inspección de equipos estáticos a la industria petrolera, ubicadas específicamente en el estado Zulia.

\section{Funciones de la gestión de seguridad industrial, ambiente e higiene ocupacional (SIAHO)}

De acuerdo con Cabrera (2002), las funciones de gestión se relacionan con la forma de administrar los procesos en las organizaciones, su éxito para alcanzar sus objetivos, y también al satisfacer sus obligaciones sociales depende, en gran medida, de sus gerentes. Si éstos realizan debidamente su trabajo es probable que la organización alcance sus metas, ya que sobre ellos cae la responsabilidad de administrar todos los recursos con los cuales cuenta la empresa. 
Como complemento el autor, explica que se ha comprobado que la eficiencia de la empresa es mucho mayor que la suma de las eficiencias de los trabajadores, y que ella debe alcanzarse mediante la racionalidad, es decir la adecuación de los medios (órganos y cargos) a los fines que se desean alcanzar; el administrador debe tener una función individual de coordinar, sin embargo parece más exacto concebirla como la esencia de la habilidad general para armonizar los esfuerzos individuales que se encaminan al cumplimiento de las metas del grupo.

Para Koontz y Weihrich (2004), las funciones de la gestión se relacionan con las actividades administrativas que ejecuta un gerente de cualquier nivel, afirma que "cuando se desempeñan como administradores, los individuos deben ejercer las funciones de planeación, organización, integración de personal, dirección y control". Es evidente que los gerentes no pueden desempeñar correctamente sus tareas si no comprenden y se muestran sensibles a los muchos elementos del ambiente externo (factores económicos, tecnológicos, sociales, políticos y éticos) que afectan a sus tareas de operación.

Desde el enfoque de Inciarte y otros (2006), están relacionados con los procesos administrativos, los cuales comprenden fases o etapas que instrumentan los cursos de acción requeridos por la gestión, a partir de los recursos y en atención a las políticas establecidas. Los procesos administrativos "se encuentran en estrecha interrelación respecto a la totalidad de acciones dirigidas al logro de los fines y objetivos de la organización.

En el caso de OIT (2011), las funciones lo refieren a directrices relativas a los Sistemas de Gestión-Seguridad y la Salud en el Trabajo (SGSST): ciclo de mejora continua, como propuesta, que incluye: Política, organización, planificaciónaplicación, evaluación y medidas para la realización de mejoras. En ese sentido la OIT (2011), explica que surge tras dos años de desarrollo y de examen internacional inter pares, las directrices relativas a los sistemas de gestión de la seguridad y la salud en el trabajo.

En este caso, la propuesta antes mencionada define la función y los elementos esenciales de un marco de gestión de la SST, tanto para los sistemas nacionales como para las organizaciones (empresas). Plantean a su vez, que en el futuro del SG-SST radicará en lograr el equilibrio adecuado entre los enfoques voluntario y obligatorio que refleja las necesidades y las prácticas a nivel local, las cuales son viables adoptarlas a cualquier tipo de empresa en lo que respecta a las relaciones laborales. Para el caso del objeto de estudio, se tomarán en cuenta los elementos propuestos por la OIT (2011), pues se identifica más con las funciones que caracterizan la gestión del SIAHO en el sector petrolero.

\section{Políticas}

Para la Norma Venezolana COVENIN 4004:2000 representa el compromiso de la organización asumido por su más alto nivel ejecutivo, frente al colectivo de sus trabajadores, la sociedad, relativo a las directrices orientadas a la conservación, el desarrollo de los recursos físicos y humanos, así como a la reducción de los daños a la salud y a los bienes.

Hernández (2005, p. 42), señalan que

“Dentro de las políticas de seguridad
está implantación y el acatamiento
de las normas de seguridad, las que
tendrán prioridad sobre todas las
normas de trabajo y producción. 
Éstas deben emerger directamente de la más alta jerarquía, darse a conocer a todos los funcionarios, técnicos y trabajadores de planta, así como reciente ingreso"

Para Muguerza (2010), las políticas en materia de seguridad industrial, son la consecuencia de la racionalidad, la filosofía y la cultura organizacional. Son reglas que se establecen para dirigir funciones y asegurar que estas se desenvuelvan de acuerdo con los objetivos deseados. Constituyen una orientación administrativa para impedir que los empleados desempeñen funciones que no desean o pongan en peligro el éxito de funciones específicas.

De acuerdo con Zúñiga (2004), como punto de partida de un sistema de gestión de seguridad, las directivas de toda compañía se deben pronunciar formalmente, a través de una política reflejando su interés por un trabajo realizado en forma segura y su compromiso hacia la salud ocupacional, posteriormente se definirán responsabilidades de todos los niveles de la organización en la implementación del programa y cumplimiento de todos los normativos que para esto haya lugar.

En el mismo orden de ideas el Instituto Nacional de los Espacios Acuáticos (INEA) (2009, p. 20), plantea que:

"La alta dirección debe definir la política que especifique claramente los objetivos generales del sistema de seguridad integral asegurándose que la misma sea adecuada al propósito de la organización, que incluye un compromiso de cumplir con los requisitos y de mejorar continuamente la eficacia del sistema. A su vez, proporciona un marco de referencia para establecer y revisar los objetivos de éste, que es comunicados y entendidos dentro de la organización y que es revisada para su continua adecuación".

Desde estas perspectivas, a criterio de la investigadora en la política de la gestión de seguridad industrial, ambiente e higiene ocupacional, debe existir un compromiso de los niveles gerenciales, pues son sus directrices que permiten hacer cumplir los objetivos del sistema de manera cuantificable y coherente. En el contexto de las empresas prestadoras de servicio de inspección de equipos estáticos a la industria petrolera, la política debe abordar por igual las preocupaciones de la dirección y de los trabajadores. Al mismo tiempo, de permitir una adecuada actividad y el reconocimiento de la seguridad e higiene ocupacional como parte integral de la gestión de la organización.

\section{Organización}

Desde ese punto de vista, de Bounds y Woods (2005, p. 15), implica "reunir y disponer con lógica las personas, equipo y otros recursos necesarios para cumplir un trabajo". La organización está íntimamente relacionada con la planeación. Un gerente que desarrolla la actividad de organización trabaja con los empleados para dividir y ordenar las tareas con el fin de cumplir los objetivos con eficiencia.

De acuerdo con Koontz y Weihrich (2004, p. 246), la organización "implica la manera como una estructura de funciones 0 puestos intencional o formalizada". A su vez, señalan que la organización consiste en la identificación y clasificación de las actividades requeridas, la agrupación de las acciones necesarias para el cumplimiento de los objetivos, la asignación de cada grupo de funciones a un administrador dotado de autoridad (delegación) necesaria para 
supervisarlo y la estipulación de coordinación horizontal y vertical, en la estructura organizacional.

Para el Sistema de Administración de la Seguridad y Salud en el Trabajo (SASST) (2005, p. 25), la organización "establecerá, mantendrá procedimientos para la identificación, medición, evaluación priorización, control continuo de los riesgos, peligros, investigación de los accidentes, enfermedades, así como la implementación de las medidas de control necesarias". A su vez, deben incluir: actividades rutinarias y no rutinarias, acciones de todo el personal que tiene acceso al sitio de trabajo (contratistas -visitantes); instalaciones y servicios en el sitio de trabajo. Asegurará que los resultados de estos análisis, así como los efectos que sean considerados cuando se establezcan y tanto las políticas como los objetivos.

En atención a los criterios expuestos, para la investigadora, la organización recae en los niveles que dirigen el curso de la empresa, con responsabilidades particulares para asegurar que el sistema de administración del mismo, sea correctamente implementado y opera conforme a los requerimientos en todas las área.

En el contexto del sector petrolero, la organización como parte de la función de la gestión permite direccionar las responsabilidades o actividades de los miembros que la conforman. Al mismo tiempo, determina a través del diseño de las funciones quien las cumplirá y a través de cuáles recursos, con la finalidad de que las actividades requeridas correspondan con el fin propuesto.

\section{Planificación}

Para Robbins y De Cenzo (2005, p. 58), la planificación comprende la "definición de los objetivos o metas de la organización, el establecimiento de una estrategia general para alcanzar estas metas y el desarrollo de una jerarquía completa de planes para integrar, coordinar actividades". Así, ésta se ocupa de los fines (lo que se tiene que hacer), al igual que de los medios (cómo se va hacer). Lo antes expuesto confirma, que todos los gerentes deben involucrarse en la planificación debido a que proporciona dirección, reduce el impacto del cambio, minimiza las pérdidas, la redundancia, y establece los estándares para facilitar el control.

Desde el enfoque de COVENIN 4001:2000, la planificación debe definir y documentar como se cumplirán los requisitos relativos en materia de prevención, ser coherente con los restantes elementos del sistema de gestión. En el caso del SIAHO, la planificación es crítica para la implantación de la política de prevención de toma eficaz. El fin de la planificación es implantar las acciones necesarias para un eficiente control de riesgos.

A este respecto, el Instituto Nacional de los Espacios Acuáticos (INEA) (2009), agrega que la planificación en materia de seguridad implica cumplir con todos los lineamientos y requisitos establecidos; mantener la integridad del sistema de seguridad cuando se implementan cambios en él. Durante el ciclo de planificación, se debe establece un plan de acción para la implantación, mejoramiento de la efectividad de cada uno de los elementos del sistema de gestión en SIAHO, considerando la complejidad y los riesgos inherentes a sus actividades, productos y / o servicios.

Finalmente, los postulados descritos permiten que la investigadora considere, que en la planificación se establezcan claramente los objetivos de la organización para cubrir el tiempo estipulado; se distribuyen a los miembros de la organización y se define la trayectoria que 
se quiere tomar para llegar a la meta, reduciendo con ello, el nivel de incertidumbre para anticiparse a los cambios.

Desde el contexto del sector petrolero, la planificación le permitirá a los involucrados mayor rendimiento de sus acciones $y$ cumplimientos de los objetivos en el tiempo fijado. Asimismo, facilita el control de los planes organizacionales, se evita cualquier desviación significativa, poco coordinada, desperdicio de tiempo, mano de obra y dinero.

\section{Evaluación}

En el caso de la OIT (2011), la evaluación implica elaborar, establecer, examinar periódicamente unos procedimientos para supervisar, apreciar y registrar con regularidad los resultados en materia de Seguridad y Salud en el Trabajo (SST). Estos procedimientos son definidos en los diferentes niveles de la gestión la responsabilidad, la rendición de cuentas y la autoridad en materia de supervisión. A su vez, involucra la investigación de las lesiones, enfermedades, dolencias e incidentes relacionados con el trabajo y sus efectos.

Como complemento, la evaluación incluye el origen y las causas subyacentes de las lesiones, enfermedades, dolencias e incidentes relacionados con el trabajo; permite identificar toda deficiencia en el sistema de gestión de la SST, estar documentada, ser llevadas a cabo por personas competentes con la participación apropiada de los trabajadores y sus representantes. Los resultados son comunicados al comité de seguridad -salud, el cual formula las recomendaciones apropiadas.

Del mismo modo, la evaluación incluye la auditoría y el examen realizado por el personal directivo. En el primer caso, se establecen disposiciones para realizar auditorías periódicas de cada uno de los elementos del sistema de gestión de la SST, con miras a determinar los resultados generales del sistema, su eficacia a la hora de proteger la seguridad, la salud de los trabajadores, de prevenir accidentes; elaborar una política, un programa de auditoría, que incluya una designación de la competencia del auditor, alcance de la auditoría, frecuencia de las auditorías, metodología de la auditoría y la presentación de informes.

En segundo lugar, referido al examen realizado por el personal directivo, para evaluar la estrategia general del sistema de gestión de la SST, a fin de determinar si cumple los objetivos previstos en materia de resultados y si atiende las necesidades en el lugar de trabajo. Los exámenes deberían basarse en los datos recopilados, en las medidas adoptadas durante el período objeto de examen, así como en la identificación de los aspectos y prioridades que deberían modificarse para mejorar los resultados y lograr los objetivos.

Desde la perspectiva de Brigelow (2006), la evaluación es una herramienta indispensable en la actividad preventiva, mediante la cual se obtiene la información precisa para determinar las decisiones apropiadas en orden a adoptar las medidas necesarias de prevención y su planificación, estableciendo las prioridades que correspondan.

Para Ruiz (2007), la evaluación abarca monitorear los peligros y riesgos para la SSO de los trabajadores de la organización los cuales deben identificarse y evaluarse sobre una base continua, debiendo aplicarse medidas de prevención-control atendiendo prioridades que tienen que considera la supresión, control del peligro/riesgo en el origen, adoptando medidas técnicas o administrativas; minimizándolo y diseñando sistemas de trabajo seguro. 
Desde el enfoque de COVENIN 4001:2000, la evaluación, es el proceso mediante el cual se obtiene la información necesaria para que la organización esté en condiciones de tomar una decisión apropiada sobre la oportunidad de adoptar acciones preventivas y en tal caso, sobre el tipo de acciones que deben adoptarse.

En consideración a los criterios establecidos, para el investigador, la evaluación es un proceso que implica tomar medidas o acciones para el reconocimiento de los factores de riesgo, prever los posibles daños y su magnitud, para poder elegir los medios para eliminarlos o minimizarlos. Por lo tanto, se trata de tomar las medidas adecuadas a tiempo. En el ámbito del sector universitario, la evaluación como parte de las funciones de la gestión, orientará el SIHAO a la organización a establecer y mantener al día sus procedimientos para identificar los riesgos, evaluarlos, controlarlos y mantener las medidas correctivas.

\section{MATERIALES Y MÉTODO}

Metodológicamente se tipificó como descriptiva, con diseño de campo, no experimental y transeccional. La población estuvo constituida por las empresas prestadoras de servicio de inspección de equipos a la industria petrolera ubicadas en el estado Zulia, siendo objeto de estudio las empresas; Alianza Sei, Sonotest y Gedinca. Tomando como informantes claves los presidentes de las empresas, los coordinadores operacionales, coordinadores SIAHO, Analistas SIAHO y los inspectores, para un total de 33 personas.

Para la recolección de datos se utilizó la encuesta mediante la aplicación de un cuestionario, el mismo quedó conformado por 12 ítems con escala de frecuencia. La validez se realizó a través del juicio de expertos, y para calcular su confiabilidad se empleó el método de Alfa de Cronbach, obteniéndose un valor de 0,96. El análisis de los datos se realizó mediante estadística descriptiva, con base en la media aritmética según el baremo establecido, mostrado en el tabla 1.

Tabla 1. Baremo para la interpretación de la media aritmética

\begin{tabular}{lcc}
\hline \multicolumn{1}{c}{ Opción de respuesta } & Rango para la media & Nivel de respuesta para la variable \\
\hline Siempre(S) & $4.21-5.00$ & Muy alta presencia \\
Casi Siempre $(\mathrm{CS})$ & $3.41-4.20$ & Alta presencia \\
Algunas Veces $(\mathrm{AV})$ & $2.61-3.40$ & Moderada presencia \\
Casi Nunca $(\mathrm{CN})$ & $1.81-2.60$ & Baja presencia \\
Nunca(N) & $1.00-1.80$ & Muy baja presencia \\
\hline
\end{tabular}

Fuente: González (2020)

RESULTADOS Y DISCUSIÓN

Con relación al indicador políticas, se evidencia según la tabla 2, un promedio de 3,11 ubicándolo en la categoría de moderada presencia. Lo cual se refleja en sus medias 3,34; 3,32 y 2,66 respectivamente, indicando que la política especifica claramente los objetivos generales del sistema de seguridad integral asegurándose que la misma sea adecuada al propósito de la empresa, se promueve las normas de seguridad hacia todos los involucrados en los procesos y se difunde por escrito la política de Seguridad y la Salud en el Trabajo a todo el personal. 
Yohanny González

Tabla 2. Indicador: Políticas

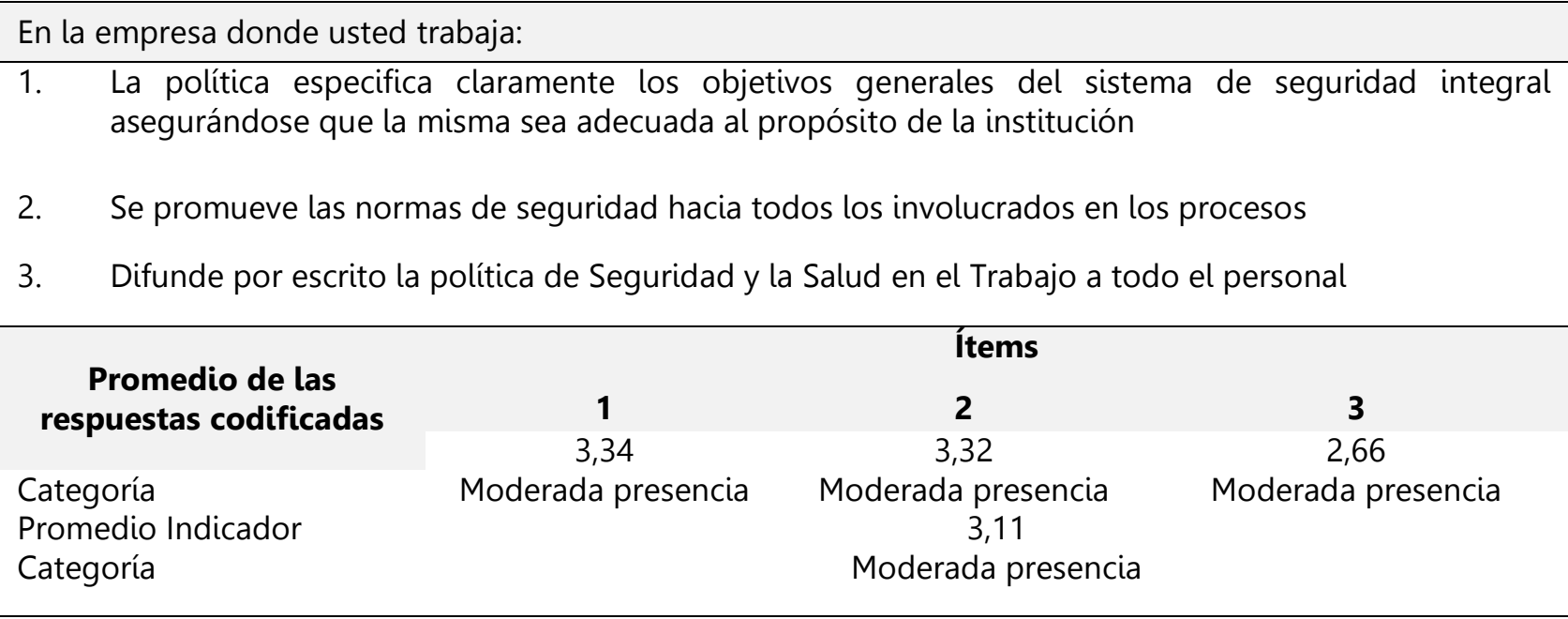

Fuente: González (2020)

Los hallazgos descritos, dan parcial validez a lo expuesto por Hernández (2005), para quien las políticas de seguridad debe estar orientada a la implantación y el acatamiento de las normas de seguridad y estas deben emerger directamente de la más alta jerarquía, y darse a conocer a todos los funcionarios, técnicos y trabajadores de planta, así como al reciente ingreso.

Los valores alcanzados, también validan parcialmente lo expuesto por la investigadora, para quien a través de la política de la gestión de seguridad industrial, ambiente e higiene ocupacional, se logra hacer cumplir los objetivos del sistema de manera cuantificable y coherente, así entonces, permite una adecuada actividad y el reconocimiento de la seguridad e higiene ocupacional como parte integral de la gestión de la organización.

En el mismo orden de ideas, se visualiza en la tabla 3 para el indicador organización un promedio de 2,74 ubicándolo en la categoría moderada presencia, indicando según los encuestados una moderada presencia de contar con procedimientos para la identificación de los riesgos $(2,75)$; de las actividades requeridas por el personal en las acciones necesarias para el cumplimiento de lo establecido $(2,72)$ y de disponer el trabajador recursos necesarios para cumplir el trabajo asignado en forma segura $(2,75)$. 
Tabla 3. Indicador: Organización

\begin{tabular}{|c|c|c|c|c|}
\hline \multicolumn{4}{|c|}{ En la empresa donde usted trabaja: } & \\
\hline 4. & \multicolumn{4}{|c|}{ Se cuenta con procedimientos para la identificación de los riesgos } \\
\hline 5. & \multicolumn{4}{|c|}{$\begin{array}{l}\text { Se identifican claramente las actividades requeridas por el personal en las acciones necesarias } \\
\text { para el cumplimiento de lo establecido. }\end{array}$} \\
\hline 6. & \multicolumn{4}{|c|}{ El trabajador dispone de recursos necesarios para cumplir el trabajo asignado en forma segura } \\
\hline \multirow{3}{*}{\multicolumn{2}{|c|}{$\begin{array}{l}\text { Promedio de las } \\
\text { respuestas codificadas }\end{array}$}} & & Ítems & \\
\hline & & & 5 & 6 \\
\hline & & & 2,72 & 2,75 \\
\hline \multicolumn{2}{|c|}{ Categoría } & & Moderada presencia & Moderada presencia \\
\hline \multicolumn{2}{|c|}{ Promedio Indicador } & \multicolumn{3}{|c|}{2,74} \\
\hline \multicolumn{2}{|c|}{ Categoría } & \multicolumn{3}{|c|}{ Moderada presencia } \\
\hline
\end{tabular}

Fuente: González (2020)

Los resultados descritos anteriormente, se corresponden parcialmente a lo establecido por el Sistema de Administración de la Seguridad y Salud en el Trabajo (SASST) (2005), que refiere que la organización debe establecer y mantener procedimientos que permitan identificar, medir, evaluar y controlar continuamente los riesgos, peligros, investigación de los accidentes, enfermedades, así como la implementación de las medidas de controles necesarias.

De igual manera, alcanzan parcialmente a validar lo establecido por la investigadora, cuando afirma que en el contexto del sector petrolero, la organización como parte de la función de la gestión permite direccionar las responsabilidades $\mathrm{o}$ actividades de los miembros que la conforman. Al mismo tiempo, determina a través del diseño de las funciones quien las cumplirá y a través de cuáles recursos, con la finalidad de que las actividades requeridas correspondan con el fin propuesto.

Para el indicador planificación, se evidencia según la tabla 4 un promedio de 3,13 ubicándolo en la categoría moderada presencia. Este resultado es producto al considerar los encuestados con un promedio de 3,02 que se clarifican las estrategias en materia de seguridad para que el personal pueda coordinar sus actividades, al mismo tiempo con un promedio de 2,64 se establecen políticas para el control de riesgos en la gestión del SIAHO y finalmente con promedio de 3.72 el trabajador tiene claramente definidos los objetivos de su puesto de trabajo. 
Yohanny González

Tabla 4. Indicador: Planificación

En la institución donde usted trabaja:

7. Se clarifican las estrategias en materia de seguridad para que el personal pueda coordinar sus actividades

8. Se establecen políticas para el control de riesgos en la gestión del SIAHO

9. El trabajador tiene claramente definidos los objetivos de su puesto de trabajo

\begin{tabular}{lccc}
\hline \multicolumn{1}{c}{ Promedio de las } & \multicolumn{1}{c}{ Ítems } \\
respuestas codificadas & $\mathbf{7}$ & $\mathbf{8}$ & $\mathbf{9}$ \\
& 3,02 & 2,64 & 3,72 \\
Categoría & Moderada presencia & Moderada presencia & Moderada presencia \\
Promedio Indicador & \multicolumn{3}{c}{3,13} \\
Categoría & \multicolumn{3}{c}{ Moderada presencia } \\
\hline
\end{tabular}

Fuente: González (2020)

Los resultados tienen parcial semejanza con el enfoque de COVENIN 4001:2000, que establece que la planificación debe definir y documentar como se cumplirán los requisitos relativos en materia de prevención, ser coherente con los restantes elementos del sistema de gestión.

De igual manera, se corresponde parcialmente con lo expuesto por la investigadora, al considerar que en la planificación se deben establecer claramente los objetivos de la organización para cubrir el tiempo estipulado; distribuir a los miembros de la organización y se definir la trayectoria que se quiere tomar para llegar a la meta, reduciendo con ello, el nivel de incertidumbre para anticiparse a los cambios.

En lo concerniente al indicador evaluación, los resultados obtenidos se recogen en la tabla 5, donde se aprecia una media de 2,67 ubicándola en una categoría de moderada presencia, indicando los encuestados que moderadamente se instituyen acciones para el control de riesgos en la gestión del SIAHO $(2,64)$; el trabajador examina periódicamente los procedimientos para registrar con regularidad los resultados en materia de SIAHO $(2,64)$ y está capacitado para identificar las deficiencias en el sistema de gestión de la Seguridad y la Salud en el Trabajo $(2,72)$.

En este sentido, se da validez parcialmente a lo expuesto en la norma COVENIN 4001:2000, que establece la evaluación como un proceso mediante el cual se obtiene la información necesaria para que la organización esté en condiciones de tomar una decisión apropiada sobre la oportunidad de adoptar acciones preventivas y en tal caso, sobre el tipo de acciones que deben adoptarse. 
Tabla 5. Indicador: Evaluación

\begin{tabular}{|c|c|c|c|c|}
\hline \multicolumn{5}{|c|}{ En la institución donde usted trabaja: } \\
\hline 10. & \multicolumn{4}{|c|}{ Se instituyen acciones para el control de riesgos en la gestión del SIAHO } \\
\hline 11. & \multicolumn{4}{|c|}{$\begin{array}{l}\text { El trabajador examina periódicamente los procedimientos para registrar con regularidad los } \\
\text { resultados en materia de SIAHO }\end{array}$} \\
\hline 12. & \multicolumn{4}{|c|}{$\begin{array}{l}\text { El trabajador está capacitado para identificar las deficiencias en el sistema de gestión de la } \\
\text { Seguridad y la Salud en el Trabajo }\end{array}$} \\
\hline \multirow{2}{*}{\multicolumn{2}{|c|}{$\begin{array}{l}\text { Promedio de las } \\
\text { respuestas codificadas }\end{array}$}} & \multicolumn{3}{|c|}{ Ítems } \\
\hline & & 10 & 11 & 12 \\
\hline \multicolumn{2}{|c|}{$\begin{array}{l}\text { Categoría } \\
\text { Promedio Indicador }\end{array}$} & $\begin{array}{l}2,64 \\
\text { Moderada presencia }\end{array}$ & $\begin{array}{c}2,64 \\
\text { Moderada presencia } \\
2,67\end{array}$ & $\begin{array}{c}2,72 \\
\text { Moderada presencia }\end{array}$ \\
\hline \multicolumn{2}{|c|}{ Categoría } & & Moderada presenc & \\
\hline
\end{tabular}

Fuente: González (2020)

Por su parte, se tiene parcial congruencia con lo expuesto por la investigadora al considerar que en el ámbito del sector petrolero, la evaluación como parte de las funciones de la gestión, debe orientar el SIHAO a la organización para establecer y mantener al día sus procedimientos a fin de identificar los riesgos, evaluarlos, controlarlos y mantener las medidas correctivas.

Como se puede apreciar en la tabla 6 , se presenta el resumen para la dimensión funciones de la gestión de SIAHO, que caracterizan la gestión de seguridad industrial, ambiente e higiene ocupacional en las empresas prestadoras de servicio de inspección de equipos estáticos a la industria petrolera, el cual muestra un valor de 2,91 ubicándose en el rango que va desde 2,61 hasta 3,40 con categoría de moderada presencia, reflejando de acuerdo a la opinión de los encuestados un conjunto de etapas que instrumentan los cursos de acción requeridos por la gestión, en tal sentido, se evidencio moderada presencia en las políticas $(3,11)$; organización $(2,74)$; planificación $(3,13)$ y evaluación $(2,67)$.

Tabla 6. Dimensión: Funciones de la gestión de SIAHO

\begin{tabular}{|c|c|c|}
\hline Indicador & Media & Categoría \\
\hline Políticas & 3,11 & Moderada presencia \\
\hline Organización & 2,74 & Moderada presencia \\
\hline Planificación & 3,13 & Moderada presencia \\
\hline Evaluación & 2,67 & Moderada presencia \\
\hline Promedio & 2,91 & Moderada presencia \\
\hline
\end{tabular}

Fuente: González (2020) 
Estos resultados concuerdan parcialmente con los fundamentos teóricos de Cabrera (2002), para quien las funciones de gestión se relacionan con la forma de administrar los procesos en las organizaciones, su éxito para alcanzar sus objetivos, y también al satisfacer sus obligaciones sociales depende, en gran medida, de sus gerentes.

Al mismo tiempo, existe parcial congruencia con lo expuesto por la investigadora, al considerar que las funciones están referidas a las directrices relativas a los Sistemas de GestiónSeguridad y la Salud en el Trabajo (SG-SST): ciclo de mejora continua, como propuesta, que incluye: Política, organización, planificaciónaplicación, evaluación y medidas para la realización de mejoras, tal como lo expresa la OIT (2011).

\section{CONCLUSIONES}

En cuanto al objetivo, en el cual se describió las funciones que caracterizan la gestión de seguridad industrial, ambiente e higiene ocupacional en las empresas prestadoras de servicio de inspección de equipos estáticos a la industria petrolera, se obtuvo que en las empresas analizadas moderadamente existe un conjunto de etapas que instrumentan los cursos de acción requeridos por la gestión.

Al detalle, se evidencio, el uso moderado de políticas de seguridad orientadas a la implantación y el acatamiento de las normas de seguridad; procedimientos que permitan identificar, medir, evaluar y controlar continuamente los riesgos; documentos que especifiquen como se cumplirán los requisitos relativos en materia de prevención que permitan obtener información necesaria para que la organización esté en condiciones de tomar una decisión apropiada sobre la oportunidad de adoptar acciones preventivas y en tal caso, sobre el tipo de acciones que deben adoptarse.

\section{REFERENCIAS}

Bounds, G. y Woods, J. (2005). Supervisión. Editorial International Thomson. México

Brigelow, J. (2006).Amenazas, riesgos y problemas ambientales. Editorial Mc GrawHill. Colombia

Cabrera, M. (2002). Programa seguridad en el trabajo. Instituto Ecuatoriano de Seguridad Social. Ecuador

Comunidad Andina de Naciones (2005). Informe de la II reunión del comité andino de autoridades en seguridad y salud en el trabajo. Disponible en: http://www.ilo.org/wcmsp5/groups/public/--ed_protect/---protrav/--safework/documents/policy/wcms_212074.pd $f$

Hernández, A. (2005) Seguridad e Higiene Industrial. Editorial Limusa. México

Inciarte, A., Marcano, N. y Reyes M. (2006). Gestión Académico-Administrativa en la Educación. Revista Venezolana de Gerencia, abril-junio, año/vol. 11, $\mathrm{n}^{\circ}$ 034, Universidad del Zulia

Instituto Nacional de los Espacios Acuáticos (INEA) (2009). Sistema de gestión de seguridad integral para operaciones portuarias. SISEINOP

Koontz, H y Weihrich, H (2004).Administración una perspectiva global. McGraw-Hill Interamericana Editores S.A Colombia

Ley Orgánica de Prevención, Condiciones y Medio Ambiente de Trabajo (LOPCYMAT) (2005).Disposiciones Fundamentales. Disponible en: http://www.inpsasel.gob.ve/moo_news/lopcy mat.htm

Muguerza, J. (2010). Gerencia de seguridad industrial. Disponible en: http://www.emagister.com/curso-gerenciaseguridad-industrial/objetivos-gerenciaseguridad-industrial 
Norma Venezolana COVENIN 4001 (2000). Sistema de Gestión de Seguridad e Higiene Ocupacional. Requisitos: Disponible en; http://www.cpzulia.org/ARCHIVOS_SSA/4001 _2000_SGSHO.pdf

Organización Internacional del Trabajo (OIT). (2011). Servicios de salud en el Trabajo (OIT). Oficina Internacional del Trabajo. Ginebra

Robbins, S. y De Cenzo (2005).Administración de los recursos humanos. Editorial Mc Graw Hill. México

Ruiz, G. (2007).Seguridad industrial. Editorial Limusa. México
Sistema de Administración de la Seguridad y Salud en el Trabajo (SASST) (2005). Programa de autogestión en seguridad y salud en el trabajo.

Disponible

en. http://autogestion.stps.gob.mx:8162/pdf/Gu \%C3\%ADa\%20de\%20Asesor\%C3\%ADa\%20S ASST.pdf

VanDer, R. (2010). La higiene ocupacional en América latina. Organización Panamericana de la Salud: Washington, D.C

Zuñiga, L. (2004). Reducción de los accidentes del trabajo mediante el cambio de la conducta hacia la seguridad. Revista Mapfre Seguridad. España 